\title{
The open-field test: An approach from multivariate analysis
}

\author{
TOSHIAKI TACHIBANA \\ Institute for Developmental Research, Aichi Prefectural Colony, Kasugai, Aichi 480-03, Japan
}

\begin{abstract}
The validity of open-field test measures was assessed by simple and multiple regression analysis, taking the difference in experience of a novel situation as the external criterion. Ambulation in the first $1 \mathrm{~min}$, rearing, latency to urination, and urination score were valid indexes of a new operational concept presented in this study, and they were also reliable. The weighting coefficients were obtained for the reduction of multiple measure scores to a single score that represents the concept.
\end{abstract}

The open-field test (OFT) has been widely used in the behavioral study of rodents. There have been conflicting results in OFT studies, and some reviews critical of the OFT have recently appeared (Archer, 1973; Royce, 1977; Walsh \& Cummins, 1976, 1978). Since "emotionality," which has been the central concept in open-field work, lacks any clear external criteria, it is difficult to assess the validity of the OFT measures as the indexes of "emotionality." It is also difficult to interpret the results of the OFT. The development of an objectively verifiable concept and the testing of the validity of the OFT measures used as its indexes are pressing needs. For this, a verifiable and significant external criterion must be established and a concept must be defined on the basis of it. In this way, the OFT results may be interpreted without ambiguity. To pursue these aims, this study assessed OFT behavior of two groups by simple and multiple regression analysis, taking the difference in experience of a novel situation as the external criterion.

There have been some studies that attempted to examine the validity of measures as indexes of " $\mathrm{emo}$ tionality" by analysis of intertrial change of openfield score (Hall, 1934; Ivinskis, 1970). In these studies, rats were exposed repeatedly to the same situation, thereby providing familiarity with them. Validity was assessed by the behavioral difference between a novel and a familiar situation. This approach, however, has a drawback in the analysis of ambulation, for it has been commonly observed that ambulation decreases with repeated exposure to a field. Thus, it might be concluded that low ambulation indicates low emotionality. The decrease in ambulation is also attributable to the decrease in exploratory behavior. It is virtually impossible to distinguish emotional ambulation from exploratory ambulation. In order to avoid this confounding, analysis should be performed on behavior in a field for which there is equal

Requests for reprints should be addressed to Toshiaki Tachibana, Institute for Developmental Research, Aichi Prefectural Colony, Kasugai, Aichi 480-03, Japan. experience among all animals. We used a method that assessed the behavioral difference in a relatively novel test situation for two groups.

\section{METHOD}

\section{Subjects}

The subjects were 73 male Sprague-Dawley rats about 9 weeks of age obtained from a colony maintained by the Institute for Developmental Research at Aichi Prefectural Colony.

\section{Apparatus}

The $60 \times 60 \mathrm{~cm}$ open field, enclosed by 60 -cm-high walls, was a 3 by 3 matrix of 20 -cm squares. The floor and walls were painted white. Illumination by overhead fluorescent lighting was $350 \mathrm{~lx}$ at the floor of the field. A ventilating fan provided background noise (about 60 phons).

\section{Procedure}

In order to group the subjects, a preliminary open-field test was administered for $3 \mathrm{~min}$. Eight measures were used: ambulation in the first $1 \mathrm{~min}$ (A1), ambulation in the total $3 \mathrm{~min}$ (A3), penetration into the inner square (PIS), rearing (RE), latency to defecation (LD), latency to urination (LU), defecation (DE), and urination score (US). If no urination or defecation occurred, a $180 \mathrm{-sec}$ latency was recorded for each latency score. The urination score was graded 0 (indicating no urination) to 3 (a large amount of urination).

The results of the preliminary test were analyzed by hierarchical cluster analysis (nearest neighbor method) (Yasuda, 1969). Based on the cluster analysis results, each rat was classified into one of two groups, equated approximately for the means of the two groups in every measure. The two groups were then assigned to either an experience (EX) group or a nonexperience (N-EX) group. In order to differentiate between the two groups on behavior in the novel situation, rats of the EX group were given "experience trials" in several novel situations at the rate of two trials on each of 6 days. In these experience trials, the rats were placed individually in the apparatus described for $10 \mathrm{~min}$ per trial: two trials in an operant conditioning chamber, a shuttle box, and a runway, and six trials in a Lashley III maze. In order to equate the amount of handling, rats of the N-EX group were given handling experience twice a day for 6 days. They were removed by hand from the home cage, held briefly, and then returned to the home cage without being placed in any apparatus. The rats of both groups were then tested in the open field for 3 min per day for 3 days. The experiments were conducted with two squads of 38 and 35 rats, and each squad was composed of an approximately equal number of rats from each of the groups. 


\section{RESULTS AND DISCUSSION}

We attributed the behavioral difference between the two groups to the difference in a factor we call "emotional reactivity to novel situation" (ERNS). ERNS is an operational concept, defined by the external criterion used in the present study. It thus differs from "emotionality." The $\mathrm{r}^{2}$ of simple regression analysis shown in Table 1 allows for a comparison with the coefficient of determination $\left(\mathbf{R}^{2}\right)$ of multiple regression analysis. The independent variable took the value of 1 or 0 . The $r^{2}$ indicates the predictability as to group difference by use of the measure (e.g., assuming a measure is $\mathrm{r}^{2}=.55,55 \%$ of the variance in a group difference is accounted for by the measure) (Kerlinger \& Pedhazur, 1973). We attributed the group difference to the difference of ERNS. Therefore, it might be argued that measures with a high value of $\mathrm{r}^{2}$ are good indicators of the ERNS; these are valid indexes of ERNS.

The resulting $\mathrm{r}^{2}$ was relatively high in $\mathrm{Al}, \mathrm{RE}$, LD, LU, and US. These five measures are valid indexes of ERNS. It is noteworthy that A3 and DE, which are the most commonly used measures of the OFT, failed to display validity. In addition, LD and LU, which have not been used as OFT measures, proved to be valid indexes. Although some studies have shown a relationship between increased levels of illumination and higher defecation and lower ambulation, others have not (Archer, 1973). This inconsistency may be due to the fact that defecation and ambulation in the total test period are not valid measures as shown by this study.

To assess the reliability of measures, the correlations between days is usually calculated. The index needed here is the reliability as to the validity of each measure. In order to examine the reliability of these $\mathrm{r}^{2}$ values, each squad was again analyzed independently. The resulting $r^{2} s$, on the 1 st day, were as follows: $\mathrm{A} 1-.20$ in the first squad, .20 in the second squad; A3-.02, .07; PIS-.01, .12; RE-.40, .47; LD-. $15, .05 ; \mathrm{LU}-.33, .25 ; \mathrm{DE}-.01, .02 ; \mathrm{US}-.14$, .14. A1, RE, LU, and US were reliable; LD and PIS were not reliable. The values of $\mathrm{A} 3$ and $\mathrm{DE}$ were too low to discuss the reliability of $\mathrm{r}^{2}$. Therefore, not only do these measures (A1, RE, LU, and US) have validity as indexes of ERNS, they also were found to be reliable. The 2 nd- and 3rd-day tests showed a trend similar to the 1st-day test in reliability. Ivinskis (1970) examined validity by varying the intensity of light and noise, and obtained results that are contradictory to those of the present study with regard to rearing and ambulation, although there was agreement in defecation. The noise level $(90 \mathrm{~dB})$ in the lvinskis study seems to be high for the rat; escape attempts from the situation might have predominated in the $90-\mathrm{dB}$ group, thereby resulting in more rearing and ambulation.

Higher predictability can be achieved by using a set of measures rather than single measures alone. Therefore, a multiple regression analysis was performed. An additional advantage of such analysis is that it is easier to interpret; when each OFT measure, such as drug effects, for example, is analyzed in isolation, interpretation of the results may be extremely difficult, since some measures show significant differences between groups, while other measures do not. This problem can be avoided by using a single score, obtained from multiple regression analysis by weighting each measure score. After obtaining a Z-score transformation for each measure, multiple regression analysis was performed with the dependent variable at 1 or 0 . This analysis amounts to a discriminant function analysis of only two groups (Kerlinger \&

Table 1

Each Measure's $r^{2}$ of Simple Regression and Means Value for Preliminary Test and Tests

\begin{tabular}{|c|c|c|c|c|c|c|c|c|}
\hline Group & A1 & A3 & PIS & $\mathrm{RE}$ & LD & $\mathbf{L U}$ & DE & US \\
\hline & \multicolumn{8}{|c|}{ Preliminary Test } \\
\hline EX & 12.40 & 35.20 & .80 & 12.10 & 7.20 & 7.70 & 4.00 & 2.00 \\
\hline N-EX & 13.00 & 37.70 & .90 & 11.90 & 7.10 & 7.90 & 4.10 & 1.90 \\
\hline \multirow[t]{2}{*}{$r^{2}$} & .01 & .02 & .00 & .00 & .00 & .00 & .00 & .00 \\
\hline & \multicolumn{8}{|c|}{ 1st Day Test } \\
\hline EX & 13.90 & 32.20 & .40 & 11.30 & 10.00 & 12.20 & 3.20 & .70 \\
\hline N-EX & 9.20 & 27.90 & .10 & 5.00 & 7.90 & 8.00 & 3.70 & 1.80 \\
\hline \multirow[t]{2}{*}{$\mathrm{r}^{2}$} & $.20^{* *}$ & .04 & $.06^{*}$ & $.43 * *$ & $.11^{* *}$ & $.29 * *$ & .01 & $.14^{* *}$ \\
\hline & \multicolumn{8}{|c|}{ 2nd Day Test } \\
\hline EX & 8.80 & 20.90 & .30 & 7.10 & 11.00 & 12.50 & 2.30 & .40 \\
\hline N-EX & 5.40 & 17.10 & .10 & 3.40 & 9.00 & 9.10 & 3.00 & 1.50 \\
\hline \multirow[t]{2}{*}{$\mathrm{r}^{2}$} & $.12^{* *}$ & .03 & .04 & $.22 * *$ & $.10^{* *}$ & $.21 * *$ & .02 & $.22 * *$ \\
\hline & \multicolumn{8}{|c|}{ 3rd Day Test } \\
\hline EX & 8.60 & 18.40 & .10 & 6.60 & 11.50 & 13.00 & 2.00 & .40 \\
\hline N-EX & 5.80 & 18.40 & .20 & 5.80 & 9.50 & 9.60 & 2.60 & 1.50 \\
\hline & $.10^{* *}$ & .00 & .03 & .01 & $.11^{* *}$ & $.28^{* *}$ & .01 & $.20 * *$ \\
\hline
\end{tabular}

Note $-L D$ and $L U$ are the square-root transformed data. $\quad{ }^{*} p<.05 . \quad *^{*} p<.01$. 
Table 2

Intercorrelations Among Eight Measures

\begin{tabular}{lrrrrrrrr}
\hline & \multicolumn{1}{c}{ A1 } & \multicolumn{1}{c}{ A3 } & PIS & RE & LD & LU & DE & US \\
\hline A1 & 1.000 & & & & & & & \\
A3 & .721 & 1.000 & & & & & & \\
PIS & .263 & .407 & 1.000 & & & & & \\
RE & .548 & .557 & .303 & 1.000 & & & \\
LD & .186 & .018 & .060 & .144 & 1.000 & & \\
LU & .269 & .054 & -.017 & .359 & .494 & 1.000 & & \\
DE & -.207 & -.108 & -.109 & -.150 & -.755 & -.296 & 1.000 & 1.000 \\
US & -.280 & -.171 & -.032 & -.303 & -.404 & -.778 & .195 & \\
\hline
\end{tabular}

Pedhazur, 1973). The results were as follows: Preliminary test, $\mathrm{R}^{2}=.04, \mathrm{~F}<1$; 1st-day test, $\mathrm{R}^{2}=.67$, $F(8,64)=16.77, p<.001 ; 2$ nd-day test, $R^{2}=.46$, $\mathrm{F}(8,64)=6.11, \mathrm{p}<.01 ; 3$ rd-day test, $\mathrm{R}^{2}=.42, \mathrm{~F}(8,64)$ $=5.76, \mathrm{p}<.01$. (Shrunken $\hat{\mathrm{R}}^{2}$ s were .63 on the 1 stday test, .39 on the 2 nd-day test, and .35 on the 3rd-day test.) The higher predictability $\left(R^{2}\right)$ was obtained by using all measures at once. Although predictability was high, $33 \%$ of all the variance-namely, $1-R^{2}$-was not accounted for by the set of eight measures in the results of the 1st-day test. This can probably be attributed to ERNS, which had not been influenced by the "experience trials," and it also resulted in an individual difference of ERNS within each group. The $\hat{R}^{2} \mathrm{~S}$ in the 2 nd- and 3 rd-day tests were low compared with those of the 1st-day test. This may be attributed to the lessened predictability of $\mathrm{A} 1, \mathrm{~A} 3, \mathrm{RE}$, and PIS, which include a factor of exploratory behavior.

Analysis should be performed on the OFT score obtained in a field that is novel to rats. Thus, analysis of regression weight (b) is focused on the results of the 1st-day test. The resulting coefficients (b)-namely, weighting coefficients-were as follows: A1, .34; A3, -.39; PIS, .16; RE, .58; LD, .33; LU, .32; DE, .35; US, .14. Because correlation among measures was relatively high (Table 2 ) and low reliability measures were involved in the analysis, it is difficult to obtain the stable regression coefficients. In addition, it is also difficult to obtain weighting coefficients ex- trapolatable to other studies by a single experiment because weighting coefficients are situation specific. Additional experiments with variations in the level of experience of the novel situation in the EX group are needed. The present approach, however, seems to be significant as a new method that meets the pressing need for an objectively verifiable concept and for a means to validate testing of the OFT measures used for its indexes.

\section{REFERENCES}

ArCHER, J. Tests for emotionality in rats and mice: A review. Animal Behaviour, 1973, 21, 205-235.

HAll, C. S. Emotional behavior in the rat: I. Defecation and urination as measures of individual differences in emotionality. Journal of Comparative Psychology, 1934, 18, 385-404.

Ivinskis, A. A study of validity of open field measures. Australian Journal of Psychology, 1970, 22, 175-183.

Kenlincier, F. N., \& Pebhazur, E. J. Multiple regression in behavioral research. New York: Hold, Rinehart \& Winston, 1973

RoycE, J. R. On the construct validity of open-field measures. Psychological Bulletin, 1977, 84, 1098-1106.

Walsh, R. N., \& Cummins, R. A. The open-field test: A critical review. Psychological Bulletin, 1976, 83, 482-504.

Walsh, R. N., \& Cummins, R. A. Caveats for future research on the open-field test: Comment on Royce. Psychological Bulletin, 1978, 85, 587-589.

Yastida, S. [Sociometrics] (in Japanese). Tokyo: Maruzen, 1969.

(Received for publication September 11, 1979; revision accepted February 7, 1980.) 